\title{
Bispectrum speckle interferometry of IRC +10216 : The dynamic evolution of the innermost circumstellar environment from 1995 to 2001
}

\author{
G. Weigelt ${ }^{1}$, Y. Y. Balega ${ }^{2}$, T. Blöcker ${ }^{1}$, K.-H. Hofmann ${ }^{1}$, A. B. Men'shchikov ${ }^{1}$, and J. M. Winters ${ }^{1}$ \\ ${ }^{1}$ Max-Planck-Institut für Radioastronomie, Auf dem Hügel 69, 53121 Bonn, Germany \\ e-mail: bloecker@mpifr-bonn.mpg.de; hofmann@mpifr-bonn.mpg.de; sasha@mpifr-bonn.mpg.de; \\ jwinters@mpifr-bonn.mpg.de \\ 2 Special Astrophysical Observatory, Nizhnij Arkhyz, Zelenchuk region, Karachai-Cherkesia 35147, Russia \\ e-mail: balega@sao.ru
}

Received 7 May 2002 / Accepted 17 June 2002

\begin{abstract}
We present new near-infrared $(J H K)$ bispectrum speckle-interferometry monitoring of the carbon star IRC+10216 obtained between 1999 and 2001 with the SAO $6 \mathrm{~m}$ telescope. The $J-, H$-, and $K$-band resolutions are 50 mas, 56 mas, and 73 mas, respectively. The total sequence of $K$-band observations covers now 8 epochs from 1995 to 2001 and shows the dynamic evolution of the inner dust shell. The present observations show that the appearance of the dust shell has considerably changed compared to the epochs of 1995 to 1998 . Four main components within a $0^{\prime} .2$ radius can be identified in the $K$-band images. The apparent separation of the two initially brightest components A and B increased from 191 mas in 1995 to 351 mas in 2001. Simultaneously, component B has been fading and almost disappeared in 2000 whereas the initially faint components C and D became brighter (relative to peak intensity). The changes of the images can be related to changes of the optical depth caused, for instance, by mass-loss variations or new dust condensation in the wind. Our recent two-dimensional radiative transfer model of IRC +10216 suggests that the observed relative motion of components A and B is not consistent with the outflow of gas and dust at the well-known terminal wind velocity of $15 \mathrm{~km} \mathrm{~s}^{-1}$. The apparent motion with a deprojected velocity of $19 \mathrm{~km} \mathrm{~s}^{-1}$ on average and of recently $27 \mathrm{~km} \mathrm{~s}^{-1}$ appears to be caused by a displacement of the dust density peak due to dust evaporation in the optically thicker and hotter environment. The present monitoring, covering more than 3 pulsation periods, shows that the structural variations are not related to the stellar pulsation cycle in a simple way. This is consistent with the predictions of hydrodynamical models that enhanced dust formation takes place on a timescale of several pulsation periods. The timescale of the fading of component B can well be explained by the formation of new dust in the circumstellar envelope.
\end{abstract}

Key words. techniques: image processing - circumstellar matter - stars: individual: IRC +10216 - stars: mass-loss - stars: AGB and post-AGB - infrared: stars

\section{Introduction}

The carbon star IRC +10216 is a long-period variable star evolving along the Asymptotic Giant Branch (AGB). It is the nearest carbon star known (distance $\sim 110-150$ pc; Crosas \& Menten 1997; Groenewegen et al. 1998) and the brightest $12 \mu \mathrm{m}$ object outside the solar system (IRAS 1986). A strong stellar wind has led to an almost complete obscuration of the star by dust. The mass-loss rate as measured in CO rotational lines amounts to $2-5 \times 10^{-5} M_{\odot} / \mathrm{yr}$ (Loup et al. 1993). Detailed two-dimensional (2D) radiative transfer modeling shows that IRC +10216 had recently suffered from an even higher mass-loss rate of $\sim 10^{-4} M_{\odot} / \mathrm{yr}$

Send offprint requests to: G. Weigelt, e-mail: weigelt@mpifr-bonn.mpg.de
(Men'shchikov et al. 2001). Based on the high mass-loss rate, long period of $P=649 \mathrm{~d}$ (Le Bertre 1992), and carbon-rich chemistry of the dust-shell, IRC +10216 is obviously in a very advanced stage of its AGB evolution (see, e.g., Blöcker 1999).

Interferometric near-infrared imaging of IRC+10216 with angular resolutions of better than 100 mas has revealed that its dust shell is clumpy and bipolar, and is changing on a time scale of only $\sim 1 \mathrm{yr}$ (Weigelt et al. 1997; Weigelt et al. 1998 [hereafter Paper I], Haniff \& Buscher 1998; Osterbart et al. 2000 [hereafter Paper II], Tuthill et al. 2000). In 1996, four components were identified in the inner dust shell of IRC +10216 within a radius of 200 mas (Weigelt et al. 1997, Paper I, Haniff \& Buscher 1998) and were denoted as A, B, C, and D in order of decreasing brightness (see Fig. 1). On larger scales the envelope of IRC +10216 appears to be spherically symmetric 
Table 1. Observational parameters. JD refers to the Julian date and $\Phi$ to the photometric phase. Phase 0 (maximum light) corresponds to $\mathrm{JD}=2449430$ (Mar. 18, 1994) as extrapolated from the light curve of Le Bertre (1992) with $P=649 \mathrm{~d} . \lambda_{\mathrm{c}}$ is the central wavelength and $\Delta \lambda$ the $F W H M$ bandwidth of the filters. $N_{\mathrm{T}}$ and $N_{\mathrm{R}}$ are the numbers of IRC +10216 speckle interferograms and reference-star speckle interferograms, respectively. $T$ is the exposure time per frame, $S$ is the seeing $(F W H M), p$ is the pixel size, and $R$ is the resolution. In the last column, the reference stars are given. The observations of 1995-1998 were already presented in Paper II and are given here for completeness.

\begin{tabular}{|c|c|c|c|c|c|c|c|c|c|c|c|c|}
\hline & Date & JD & $\Phi$ & $\begin{array}{c}\lambda_{\mathrm{c}} \\
{[\mu \mathrm{m}]}\end{array}$ & $\begin{array}{c}\Delta \lambda \\
{[\mu \mathrm{m}]}\end{array}$ & $N_{\mathrm{T}}$ & $N_{\mathrm{R}}$ & $\begin{array}{c}T \\
{[\mathrm{~ms}]}\end{array}$ & $\begin{array}{c}S \\
{\left[{ }^{\prime \prime}\right]}\end{array}$ & $\begin{array}{c}p \\
{[\mathrm{mas}]}\end{array}$ & $\begin{array}{c}R \\
{[\mathrm{mas}]}\end{array}$ & Ref. star \\
\hline \multirow[t]{2}{*}{$J$} & 2 Apr. 1996 & 2450176 & 1.15 & 1.24 & 0.28 & 1196 & 981 & 200 & 1.2 & 14.6 & 149 & HIP 51133 \\
\hline & 10 Mar. 2001 & 2451979 & 3.93 & 1.24 & 0.14 & 1042 & 783 & 160 & 1.0 & 13.3 & 50 & HD 83871 \\
\hline \multirow[t]{2}{*}{$H$} & 23 Jan. 1997 & 2450472 & 1.61 & 1.64 & 0.31 & 1665 & 2110 & 100 & 1.5 & 19.8 & 70 & HIP 52689 \\
\hline & 10 Mar. 2001 & 2451979 & 3.93 & 1.65 & 0.32 & 607 & 915 & 30 & 1.0 & 20.1 & 56 & HD 83871 \\
\hline \multirow[t]{8}{*}{$K$} & 8 Oct. 1995 & 2449999 & 0.88 & 2.12 & 0.02 & 251 & 266 & 100 & 1.5 & 31.5 & 92 & SAO 116569 \\
\hline & 3 Apr. 1996 & 2450177 & 1.15 & 2.17 & 0.33 & 1403 & 1363 & 70 & 2.5 & 14.6 & 82 & HIP 51133 \\
\hline & 23 Jan. 1997 & 2450472 & 1.61 & 2.19 & 0.41 & 2165 & 1539 & 50 & 0.9 & 30.6 & 87 & HIP 52689 \\
\hline & 14 Jun. 1998 & 2450979 & 2.39 & 2.17 & 0.33 & 800 & 571 & 50 & 1.6 & 30.6 & 87 & HIP 50792 \\
\hline & 3 Nov. 1998 & 2451121 & 2.61 & 2.20 & 0.20 & 1087 & 842 & 40 & 1.3 & 27.2 & 75 & HIP 49583 \\
\hline & 24 Sep. 1999 & 2451446 & 3.11 & 2.12 & 0.21 & 2702 & 1383 & 80 & 0.9 & 26.4 & 73 & HIP 49637 \\
\hline & 15 Oct. 2000 & 2451833 & 3.70 & 2.09 & 0.02 & 1740 & 2091 & 30 & 1.3 & 26.8 & 73 & HIP 49637 \\
\hline & 9 Mar. 2001 & 2451978 & 3.93 & 2.09 & 0.02 & 390 & 777 & 20 & 1.0 & 27.0 & 73 & 31 Leo \\
\hline
\end{tabular}

(Mauron \& Huggins 1999, 2000). Since most dust shells around AGB stars are known to be spherically symmetric on larger scales, whereas most proto-planetary nebulae (PPN) appear in axisymmetric geometry (Olofsson 1996), it is likely that IRC +10216 has already entered the transition phase to PPN. This suggests that the break of symmetry already takes place at the end of the AGB evolution. So far, only a few AGB objects are known to show prominent asphericities of their dust shells in the near-infrared, and are therefore believed to have entered this transition phase at the end of their AGB life. This includes, for instance, the carbon star CIT6 (Monnier et al. 2000), and the oxygen-rich stars AFGL 2290 (Gauger et al. 1999) and CIT 3 (Hofmann et al. 2001).

Recently, we have developed a 2D radiative transfer model of IRC +10216 (Men'shchikov et al. 2001; hereafter Paper III) which can explain many aspects of the nebula. In this model, the star is located at component B and is surrounded by an optically thick shell with bipolar cavities. The brightest southern component $\mathrm{A}$ is identified with radiation emitted and scattered in the optically thinner southern cavity of the dense shell. However, Weigelt et al. (1998), Haniff \& Buscher (1998), and Tuthill et al. (2000) argued that the star is located at the position of component $\mathrm{A}$ and that components $\mathrm{B}, \mathrm{C}$, and $\mathrm{D}$ are dust clouds. In the present paper, we attempt to explain the recent changes in the nebula in light of the 2D model of Paper III, and will comment on the alternative interpretations only in passing.

High-resolution near-infrared monitoring of the components $\mathrm{A}, \mathrm{B}, \mathrm{C}$, and D has already revealed that the dust shell of IRC +10216 is rapidly evolving. The $6 \mathrm{~m}$ telescope speckle observations presented in Paper II cover five epochs between 1995 and 1998 and show that the separations between the different components had steadily been increasing. For example, the distance between the initially brightest components $\mathrm{A}$ and $\mathrm{B}$ increased by $36 \%$ during $1995-1998$. These results are in very good agreement with Keck telescope $K$-band observations obtained with the highest resolution to date at 7 epochs between 1997 and 1999 by Tuthill et al. (2000). Such direct observations of the dust-shell evolution offer an ideal opportunity to study the mass-loss process in the late stages of AGB evolution, revealing details of the dust formation process as well as the geometry and clumpiness of the stellar wind.

This paper presents new near-infrared bispectrum speckle interferometry monitoring of IRC +10216 obtained with the SAO $6 \mathrm{~m}$ telescope in 1999-2001, adding 3 new epochs to the already available data of dust-shell evolution. The present observations show that the appearance of the dust shell has considerably changed compared to the epochs of 1995 to 1998. This paper is organized as follows. In Sect. 2, $K$-band observations of the dust-shell evolution from 1999 to 2001, a comparison of $J_{-}, H$-, and $K$-band images of 1996/97 and 2001, and $J-H, J-K$, and $H-K$ color images are presented. In Sect. 3, these observations are discussed on the basis of the general morphology, 2D radiative transfer models, and dust-formation models. Conclusions are given in Sect. 4.

\section{Observations}

\subsection{Data reduction}

New bispectrum speckle-interferometry observations of IRC +10216 were carried out with the Russian $6 \mathrm{~m}$ telescope at the Special Astrophysical Observatory in September 1999, October 2000, and March 2001. The speckle interferograms were recorded with our HAWAII speckle camera in the $J, H$, and $K$ bands. The speckle transfer function was derived from speckle interferograms of unresolved reference stars. Table 1 lists the observational parameters of these measurements 


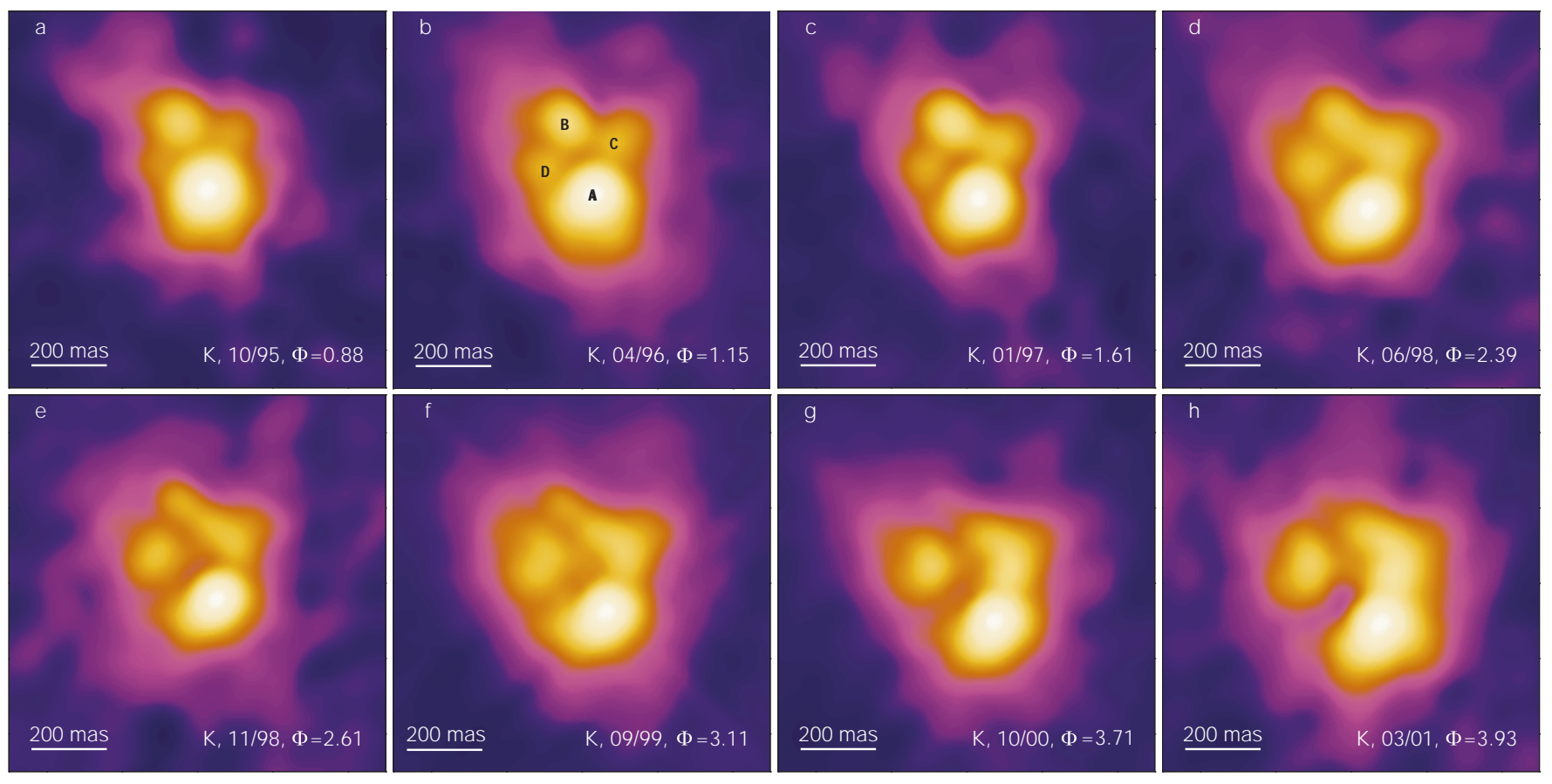

Fig. 1. $K$-band speckle reconstructions of IRC +10216 for 8 epochs from 1995 to 2001 . The total area is $1^{\prime \prime} \times 1^{\prime \prime}$. All images are normalized to the brightest pixel and are presented with the same color table. North is up and east is to the left.
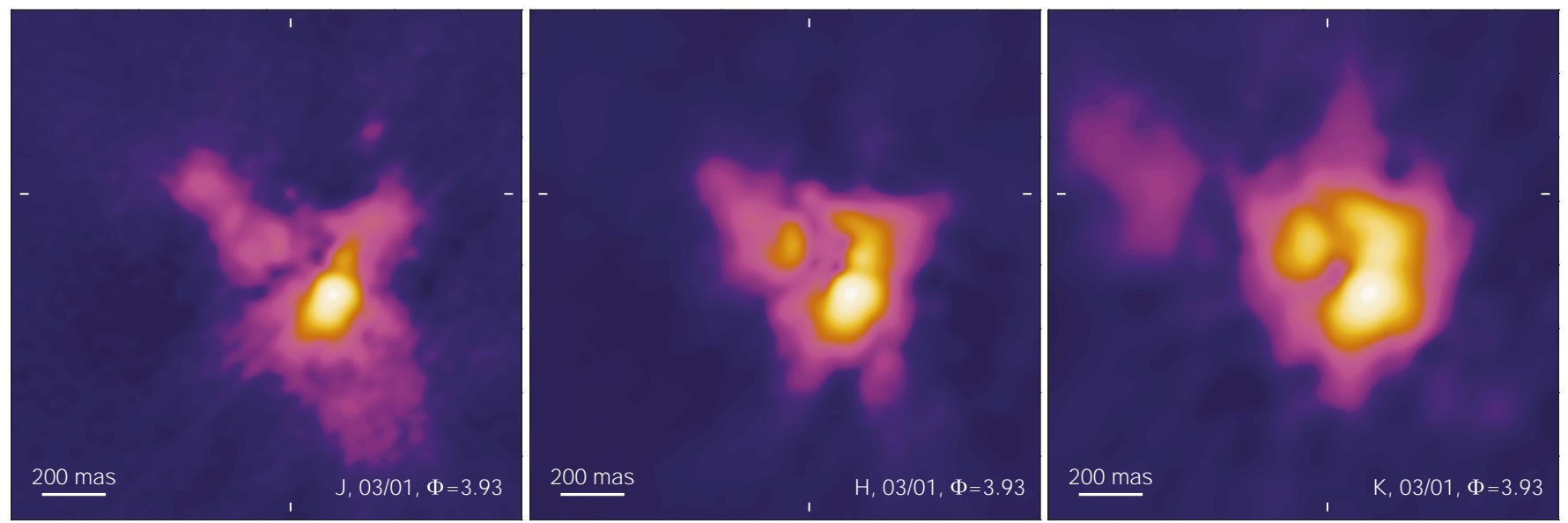

Fig. 2. $J$-, $H$ - and $K$-band speckle reconstructions of IRC +10216 in March 2001 . The total area is $1.6^{\prime \prime} \times 1.6^{\prime \prime}$. All images are normalized to the brightest pixel and are presented with the same color table. North is up and east is to the left. The tick marks indicate the likely position of the central star.

(1999-2001) complemented with the data of Paper II (19951998). Images of IRC +10216 with resolutions of 50 mas $(J), 56 \mathrm{mas}(H)$, and 73 mas $(K)$ were reconstructed from the speckle interferograms using the bispectrum speckleinterferometry method (Weigelt 1977; Lohmann et al. 1983; Hofmann \& Weigelt 1986). The modulus of the object Fourier transform (visibility function) was determined with the speckle interferometry method (Labeyrie 1970). The $H$ - and $K$-band images are diffraction-limited, the resolution in $J$ is only slightly (14\%) below the diffraction limit. Together with the data of Paper II, a time series of $8 \mathrm{~K}$-band images, taken between 1995 and 2001, is now available. Additionally, two epochs are covered in $J(1996,2001)$ and $H(1997,2001)$. Figures 1-3 show the reconstructed $K$-band images of the innermost region of IRC +10216 for all 8 epochs between 1995 and 2001, the most recent $J, H$, and $K$ images of March 2001, and a comparison of the $J, H$, and $K$ reconstructions from 1996/97 and 2001.

\subsection{Evolution in the $K$ band from 1995 to 2001}

The $K$-band images (Fig. 1) show several compact components within a 200 mas radius. We denote these components as A, B, C, and D in the order of decreasing brightness in the 1996 image (cf. Paper I). As already shown in Paper II, the dust shell is dynamically evolving and changes its shape on a timescale of $\sim 1$ yr. For instance, the apparent separation of the two initially brightest components A and B increased from 191 mas 


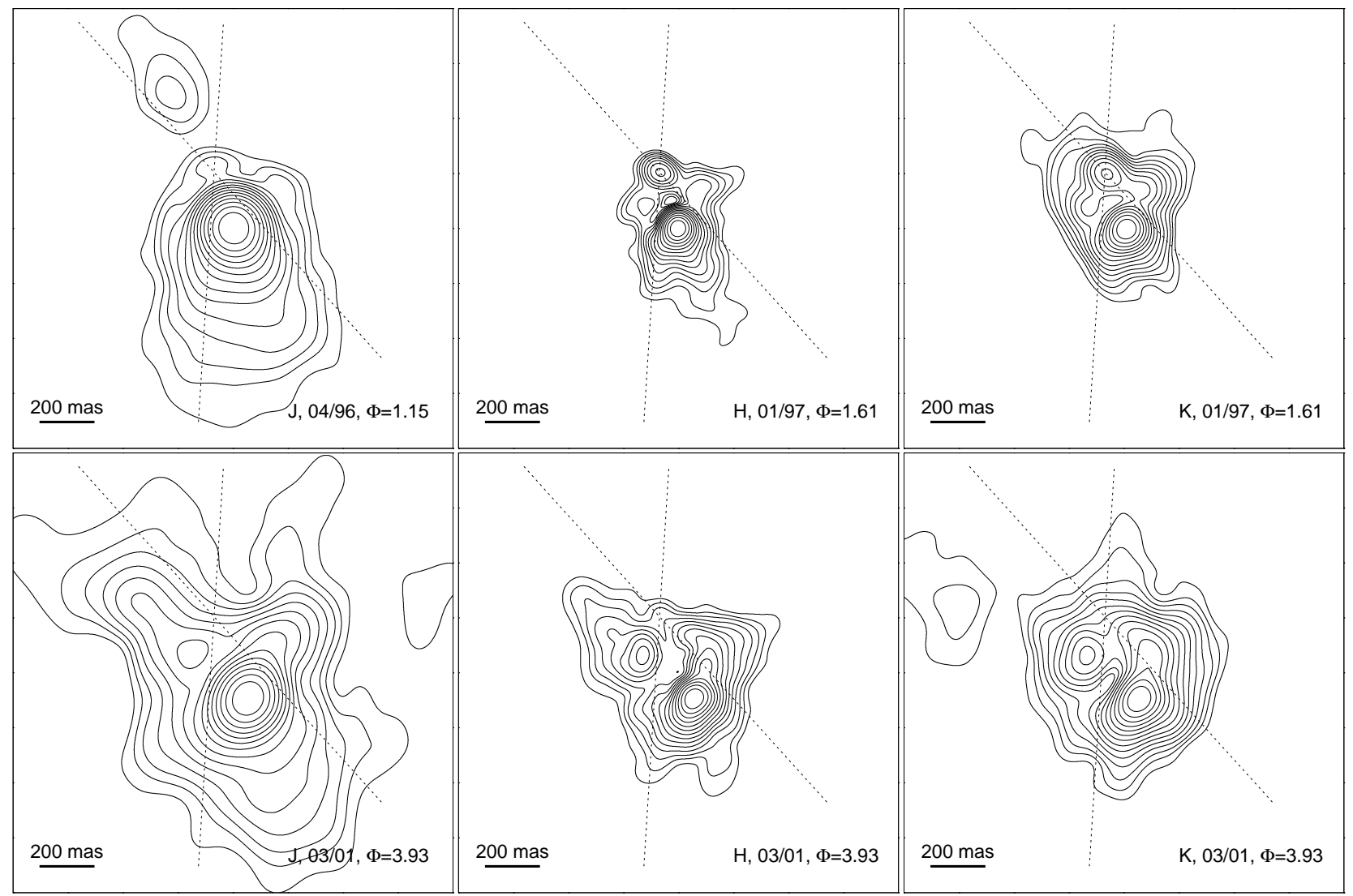

Fig. 3. Left column: J-band speckle images of IRC +10216 in April 1996 (top) and March 2001 (bottom) reconstructed with the same resolution of 149 mas. Middle column: H-band speckle images of IRC +10216 in January 1997 (top) and March 2001 (bottom) reconstructed with the same resolution of 73 mas. Right column: K-band speckle images of IRC +10216 in January 1997 (top) and March 2001 (bottom) reconstructed with the same resolution of 90 mas. Contour lines are shown from 0.3 mag to 4.2 mag relative to the peak brightness in steps of 0.3 mag. North is up and east is to the left. The dashed lines indicate the biconical geometry of the cavities according to the 2D radiative transfer model of Paper III; the lines intersect at the position of component B.

in 1995 to 351 mas in 2001. At the same time, B has faded and almost disappeared in 2000 whereas the initially faint components $\mathrm{C}$ and D have become brighter. In 2001, the intensity level of $\mathrm{C}$ increased to almost $40 \%$ of the peak intensity of $\mathrm{A}$. The components $\mathrm{A}$ and $\mathrm{C}$ appear to have started merging in 2000 .

During these six years, the compact and bright core of component A has been changing its shape continuously. While it was almost spherically symmetric in 1995 , it is getting more elongated in the following years. For example, the ratio of minor to major axis drops to $\sim 0.77$ during the years 1998 and 1999 , with the major axis being aligned along the position angle $-48^{\circ}$ (measured at $35 \%$ intensity level). The elongation of the inner core of A stays roughly constant. Additionally, the morphology of the image in 2001 gives evidence of the potential development of a bulge-like structure at $\mathrm{PA}=-123^{\circ}$ relative to the center of $\mathrm{A}$, which may indicate the birth of a new component.

The apparent separations of the various components with respect to A and B are shown in Fig. 4 as a function of time. The separations refer to the intensity maxima of the respective components which evolve along approximately constant position angles in the 1990s. Later, in 2000 and 2001, the position angles change moderately.

During the first 5 epochs the apparent separation between $\mathrm{A}$ and $\mathrm{B}$ increased by 23 mas/yr (Paper II). Interpreting this increase as real motion would lead to $14 \mathrm{~km} \mathrm{~s}^{-1}$ within the plane of the sky (for a distance of $130 \mathrm{pc}$ ). The corresponding leastsquares fit of the 1995-2001 data gives $28 \mathrm{mas} / \mathrm{yr}\left(18 \mathrm{~km} \mathrm{~s}^{-1}\right)$. After 1999 component B became very faint and almost disappeared in 2001. Therefore, its position in 2000 and 2001 is subject to larger uncertainties. It should be noted that the complete series from 1995 to 2001 can be best matched by a parabolic fit. This may suggest an apparent acceleration of $\sim 5 \mathrm{mas} / \mathrm{yr}^{2}$. The fit is shown in Fig. 4. A similar acceleration term of $\sim 3 \mathrm{mas} / \mathrm{yr}^{2}$ was inferred by Tuthill et al. (2000) from Keck telescope $K$-band data for 7 epochs from 1997 to 1999.

The positions of component $\mathrm{D}$ with respect to $\mathrm{A}$ are of particular interest since they are well defined for the complete data set: whereas B considerably fades and C appears to start merging with the bright core, component $\mathrm{D}$ brightens and is well separated from A in all images. In Paper II, we derived a velocity of $21 \mathrm{mas} / \mathrm{yr}\left(13 \mathrm{~km} \mathrm{~s}^{-1}\right)$ for the increase of the apparent separation A-D during 1995-1998. With the new data we obtain $18 \mathrm{mas} / \mathrm{yr}\left(11 \mathrm{~km} \mathrm{~s}^{-1}\right)$ for 1995-2001, as shown in Fig. 4. 
For the evolution of these components, there is no evidence of acceleration. The distance A-C has only slightly changed during the 6 years. A linear regression yields 5 mas/yr for 19951999. After 1999, A and C started merging and the separations do not follow the above relation anymore (see Fig. 4).

In our radiative transfer modeling (Paper III), component A was identified with the southern cavity of a bipolar structure, whereas the location of the central star is at component $\mathrm{B}$. In the lower panel of Fig. 4 we therefore present the apparent separations of the components $\mathrm{C}$ and $\mathrm{D}$ with respect to component $\mathrm{B}$. Least-squares fits give $8 \mathrm{mas} / \mathrm{yr}\left(5 \mathrm{~km} \mathrm{~s}^{-1}\right.$, for 1995-1999) for the separation increase of B-C and 9 mas/yr $\left(6 \mathrm{~km} \mathrm{~s}^{-1}\right)$ for that of B-D. Due to the merging of A and C, the separation B-C increases strongly in 2000 and 2001. The position of B becomes ill-defined in the later epochs due to its dimming.

\subsection{Changes of the JHK images from $1996 / 97$ to 2001}

Figure 2 shows the $J, H$, and $K$ images of IRC +10216 obtained in March 2001. The resolution in $H$ and $K$ is diffraction-limited (56 and 73 mas, respectively) whereas in $J 86 \%$ of the diffraction limit is reached (50 mas). While the brightness of the inner dust shell components (yellow regions) increases with increasing wavelength, the brightness of the faint surrounding nebula (red regions) stays roughly constant. In the $J$ image, faint nebular structure on sub-arcsecond scales can be seen. The northeastern arm $\left(\mathrm{PA} \sim 51^{\circ}\right.$ with respect to component $\left.\mathrm{A}\right)$ appears to have a clumpy structure. Various components can be identified in this structure at different separations from the center. The innermost clump is located at a separation of 256 mas from the center of A, almost coinciding with the position of component $\mathrm{D}$ in the $H$ and $K$ images. A closer inspection reveals that this clump, in turn, resolves into two sub-structures of similar size. Beyond this double knot, two further clumps can be identified at a separation of 378 mas and 522 mas, resp. The inner bright core A appears to show a multi-component structure in the $J$ band as well. It consists of two components separated by $\sim 60$ mas at $\mathrm{PA} \sim 150^{\circ}$.

Figure 3 shows the $J$-, $H$-, and $K$-band images of IRC +10216 in $1996 / 97$ compared to those in 2001. For a given band, the images are reconstructed with the same resolution ( 149 mas in $J, 73$ mas in $H$, and 90 mas in $K$ ). The dustshell morphology and evolution observed in $H$ are very similar to those in $K$. For instance, the apparent separation A-D increased from 163 mas in 1997 to 245 mas in 2001, at the same time component A became elongated (axis ratio of 0.65 in 2001) and a bridge between $A$ and $C$ has formed. In the $J$-band, where one predominantly observes scattered light, the images changed significantly as well. As in the other bands, component A became elongated along the same position angle. Three arms can be identified in north-western, north-eastern and south-western direction (see also Fig. 2). The appearance of the northern arms becomes much more prominent between the two epochs.

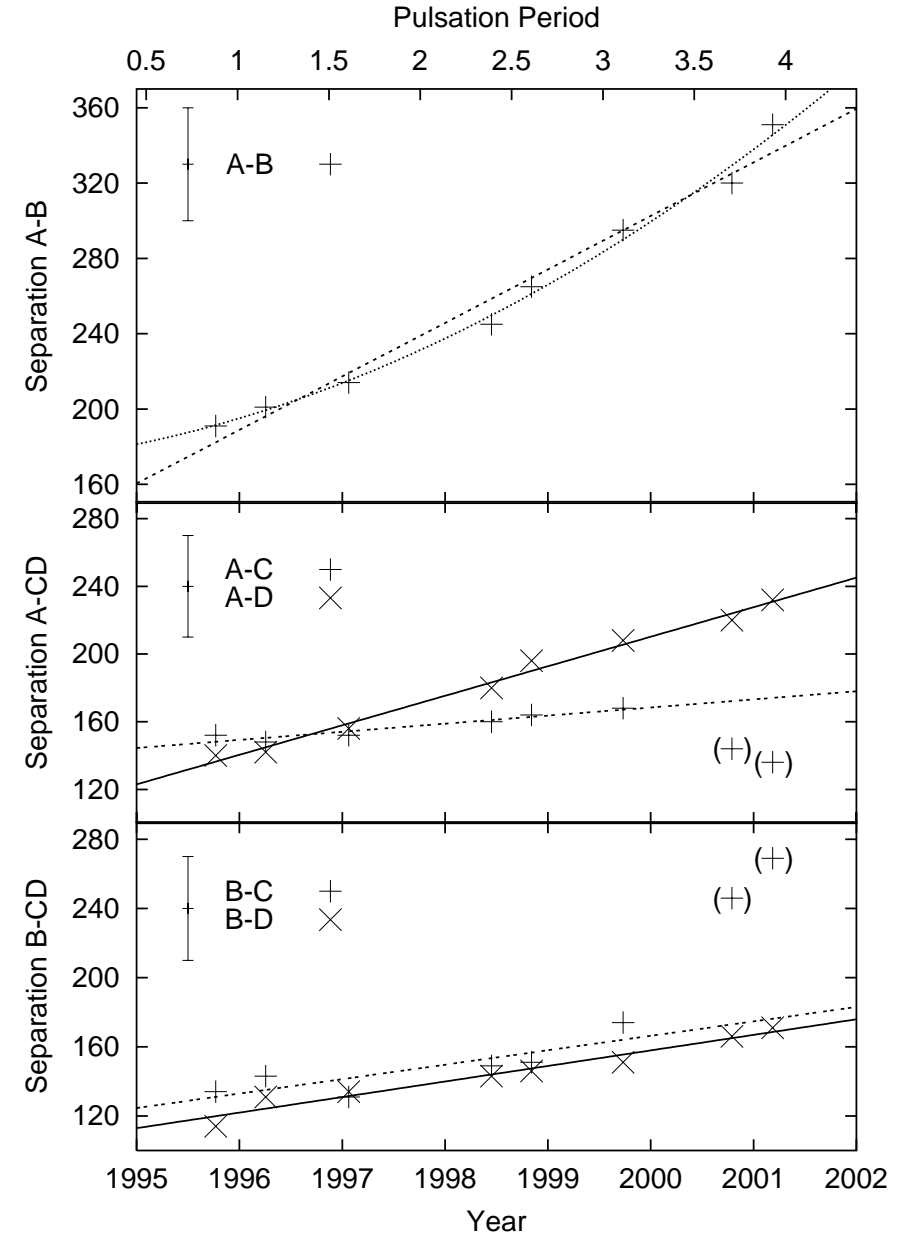

Fig. 4. Apparent separation of the components A, B, C, and D with respect to $\mathrm{A}$ and $\mathrm{B}$ as function of time and photometric phase, resp. The lines give least-squares fits of the 1995-2001 data resulting in average velocities of $28 \mathrm{mas} / \mathrm{yr}$ (A-B), $5 \mathrm{mas} / \mathrm{yr}$ (A-C, for 1995-1999), 18 mas/yr (A-D), 8 mas/yr (B-C, for 1995-1999), and 9 mas/yr (B-D). The statistical velocity error bars amount to \pm 1 mas/yr (A-C, A-D, B-D), \pm 2 mas/yr (A-B), and \pm 3 mas/yr (B-C). The separations $\mathrm{A}-\mathrm{C}$ and $\mathrm{B}-\mathrm{C}$ in 2000 and 2001 (shown in parentheses) are not well-defined due to the merging of $\mathrm{A}$ and $\mathrm{C}$. Also shown is a parabolic fit of the apparent increase of A-B corresponding to an acceleration of $\sim 5 \mathrm{mas} / \mathrm{yr}^{2}$. Note, however, that the existence of such acceleration terms is not very certain judged from the present data. The error bar given by the pixel size (cf. Table 1) is shown in the upper left corner of each panel.

\subsection{J-K and H-K color images}

To compute color images, the $J, H$, and $K$ images observed in 2001 were reconstructed with the same resolution of 115 mas. In our previous study (Paper II) we found that at previous epochs, when both components A and B were clearly visible, the peak separations in the $J, H$, and $K$ images were the same within a few milli-arcseconds (at the same epochs). Therefore, we obtained almost identical color images independently of whether the images were centered on component A or B. The color images are not very sensitive to relative shifts within some milli-arcseconds. 
The resulting $J-K$ and $H-K$ color images for the epoch of March 2001 are shown in Fig. 5 together with the intensity contour lines in the $K$ band. The color maps are confined to regions which are brighter than $2 \%$ of the peak intensity in the $J$ and $K$ images $(J-K)$ and $3 \%$ of the peak intensity in the $H$ and $K$ images $(H-K)$, resp. The absolute magnitudes were calibrated with coeval photometry kindly provided by B. Yudin. The integrated magnitudes in $J, H$, and $K$ (aperture $10^{\prime \prime}$ ) are $5.98 \mathrm{mag}, 3.51 \mathrm{mag}$, and $0.52 \mathrm{mag}$, resp. In Fig. 5, white color refers to magnitude differences of $7.4 \mathrm{mag}(J-K)$ and $3.9 \mathrm{mag}(H-K)$; the darkest grey refers to magnitude differences of $5.0 \mathrm{mag}(J-K)$, and $2.7 \mathrm{mag}(H-K)$. As in 1997 (Paper II), component A turned out to be clearly the bluest part of the inner dust-shell region, as expected for a cavity illuminated by scattered light. Note that the star is obscured by an optically very thick dust shell ( $\tau_{V} \approx 40$, cf. Paper III) and therefore it must be located within the red area of the color maps.

\section{Discussion}

\subsection{Dust and winds}

On larger scales, most AGB stars are surrounded by dust shells of spherical symmetry. This holds also for the dust shell of IRC +10216 (Mauron \& Huggins 1999, 2000). However, on sub-arcsecond scales this symmetry appears to break down for giants in very advanced stages of their AGB evolution, with IRC +10216 being their most prominent representative. The $2 \mathrm{D}$ radiative transfer modelling presented in Paper III strongly suggested that the central star of IRC +10216 is surrounded by an optically thick dust shell with bipolar cavities of a full opening angle of $36^{\circ}$ tilted with the southern lobe towards the observer (although there may exist different interpretations, see Sect. 1). The bright compact component $\mathrm{A}$ is not the direct light from the central star but is the southern cavity of this bipolar structure dominated by scattered light. According to this model, the carbon star is at the position of the fainter northern component B.

This dust-shell model and the possible existence of acceleration within the cavities may be understood in terms of the beginning operation of interacting winds which later lead to the shaping of planetary nebulae (Kwok 1982; Balick 1987). Within this scenario a fast stellar wind interacts with the fossil, slow AGB wind. The AGB mass-loss is thought to be aspherical and to take preferentially place in the equatorial plane. Accordingly, the polar regions show lower densities than the equatorial regions. Later, after a fast wind has developed, the less dense polar material is carved out leading to the formation of outflow cavities. This fast wind can be expected to turn on slowly at the very end of the AGB evolution resulting into the formation of biconical dust-shell structures on subarcsecond scales as observed for IRC +10216 .

The high spatial and temporal resolution of the present observations reveals details of the mass-loss process even in the immediate vicinity of the dust condensation zone. The monitoring, covering more than 3 pulsation periods, shows that the structural variations are not related to the stellar pulsation cycle in a simple way. This is consistent with the predictions of hydrodynamical models that enhanced dust formation takes place on a timescale of several pulsation periods (Fleischer et al. 1995). In Sect. 3.3 simplified dust formation models are compared with the observations.

\subsection{Observed changes interpreted by $2 D$ modeling}

The large number of epochs available now seems to make the interpretation of the sub-arcsecond structures and their evolution even more difficult and puzzling than before. Here we suggest a physical picture of what presently is going on in this object based on the results of our previous 2D radiative transfer modeling (Paper III). The model was designed to derive the structure and physical properties of the star and its dusty envelope at a single moment, corresponding to the third epoch (January 23, 1997) of our images. Our simplified attempt to handle the difficult problem of self-consistent time-dependent multidimensional radiative transfer modeling will be presented elsewhere (Men'shchikov et al. 2002, submitted).

\subsubsection{The star and its evolving environment}

Key point to the understanding of the structure and evolution of the sub-arcsecond environment of IRC +10216 is the knowledge of the position of the central star in the images. As we have suggested in the previous modeling (Paper III), the brightest compact peak A seen in all images is not the direct light from the central star. The star is most likely located at the position of the fainter peak $\mathrm{B}$, whereas component $\mathrm{A}$ is the radiation emitted and scattered in the optically thinner conical cavity of the optically thick bipolar dust shell. The even fainter components $\mathrm{C}$ and $\mathrm{D}$ in the $H$ and $K$ images were identified with smaller-scale deviations of the density distribution of the circumstellar environment from axial symmetry (Figs. 1 and 2 in Paper III). These inhomogeneities are less opaque than other, more regular regions of the compact dense shell.

An alternative interpretation of the morphology of our images of IRC +10216 would be that components $\mathrm{A}$ and $\mathrm{B}$ are the lobes of a bipolar nebula and that the star is located in the dark region between them. However, this interpretation disagrees with our radiative transfer modeling presented in Paper III. A reason for this is that the optically thick dust which would form the bipolar lobes cannot exist so close to the star, at half the distance between the components. Thus, the star is probably located either at A or B. The fan-shaped morphology of component $\mathrm{A}$ in the $J$ - and $H$-band images, as well as other evidence presented in Papers II and III, strongly suggest that the star is located at the position of B.

Having defined the stellar position at component B, we can now interpret the evolving appearance of IRC +10216 in terms of an increased mass-loss rate during the last $\sim 30$ years. One of the main features of the entire 8-epoch sequence of imaging is the relative fading of the stellar component $\mathrm{B}$ (Figs. 1 and 2). Other changes seen in the images are the increasing distances of components A, C, D from the star (Fig. 4) and their greatly varying shapes. Our radiative transfer modeling (Paper III) has shown that the observed components are 


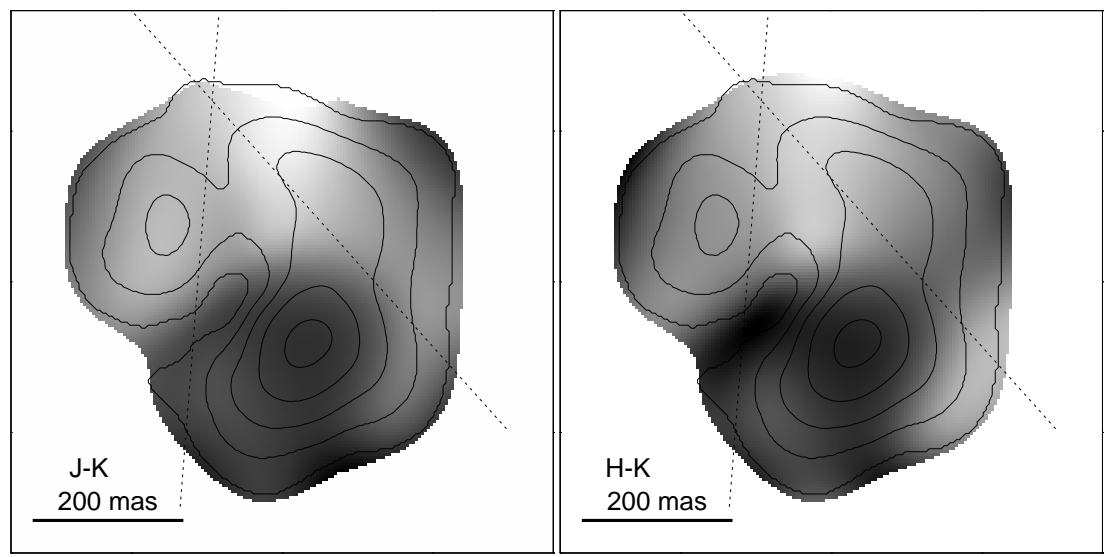

Fig. 5. $J-K$ (left) and $H-K$ (right) color images (greyscale images) of the central region of IRC +10216 in March 2001, confined to regions which are brighter than $2 \%$ of the peak intensity in the $J$ and $K$ images ( $J-K$ color image) and 3\% of the peak intensity in the $H$ and $K$ images $(H-K$ color image), resp. The resolution is 115 mas. Contour lines refer to the $K$-band image and are shown from 0.2 mag to 3 mag relative to the peak brightness in steps of 0.7 mag. To construct the respective color images, the $J, H$, and $K$ reconstructions were centered at the positions of maximum intensity. White regions refer to magnitude differences of 7.4 mag $(J-K)$ and 3.9 mag $(H-K)$; the regions shown in the darkest grey correspond to magnitude differences of $5.0 \mathrm{mag}(J-K)$ and $2.7 \mathrm{mag}(H-K)$. North is up and east is to the left. Dashed lines indicate the biconical geometry of the cavities according to the $2 \mathrm{D}$ radiative transfer model of Paper III.

cavities in the dense opaque shell; therefore, the observed motion requires an additional discussion (Sect. 3.2.4).

\subsubsection{The near-IR images and model cavities}

In order to better visualize the relative location of the components, we have shown the structure (conical cavities) of our model (Paper III) in the observed images by dashed lines in Figs. 3 and 5. The lines intersecting at the position of the central star and making an angle of $46^{\circ}$ between them, outline the biconical geometry of the cavities.

The April $1996 J$-band image in Fig. 3 best exhibits the bipolar geometry of IRC +10216 . Clearly visible at this wavelength are the fan shape of the brightest component $\mathrm{A}$ and even the scattered light from the opposite (northern) cavity on the far side of the dense shell. Faint direct stellar light is visible near the origin of the conical cavities. The star (component B) appears much brighter in the January $1997 \mathrm{H}$-band image (Fig. 3), whereas the fan shape of the cavity (component A) is less prominent but still well visible at this wavelength. The $H$ and $K$ images in 1997 agree very well with our model presented in Paper III. The March $2001 H$ and $K$ images are qualitatively similar to the older images obtained in 1997, but distorted by the appearance of several fainter structures close to component $\mathrm{D}$ and by merging of the bright component A with component C. The faint direct stellar light has become more difficult to identify in $H$ and it has been buried in the enhanced dust emission from all the other components in the March $2001 \mathrm{~K}$-band image.

The most recent $J$ image of IRC +10216 with a 3 times higher resolution (50 mas, Fig. 2) shows in much greater detail the fine structure of the envelope. The image reveals a rather isolated, faint peak at the position of the central star (marked in Fig. 2). The following evidence suggests that this peak is indeed the direct light from the star, not just a clump that happened to be there: (1) its position angle relative to $\mathrm{A}(\mathrm{PA} \approx$ $20^{\circ}$ ) is the same as in all the images where the star (component B) is clearly visible, (2) the $H$ image at the same epoch shows a (less isolated) peak at the same position, (3) the distance of approximately 347 mas between the peak and the component A is consistent with the fit of Fig. 4.

One might think that the faint stellar peak should be much better visible in $K$ band (Fig. 2), where optical depths are significantly lower compared to $J$. However, the greatly enhanced hot dust emission in $K$ band may well make it completely invisible. In fact, from the continuum of the model stellar atmosphere used in Paper III, we estimate that the stellar brightness in $J$ and $K$ bands is approximately the same. The dust model of Paper III would predict that the star is a factor of $\sim 1.8 \times 10^{3}$ brighter in $K$ than in $J$ band, whereas our new model of IRC+10216 (Men'shchikov et al., submitted), computed specifically for the latest epoch of March 2001, predicts a larger factor of $\sim 1.3 \times 10^{4}$. On the other hand, from the calibrated color images of Fig. 5 we know that the nebula becomes much brighter between $J$ and $K$ (and thus redder), by a factor of $\sim 1.4 \times 10^{4}$. Therefore, it is unlikely that in $K$ the stellar peak is visible better than in $J$ band.

\subsubsection{Shapes of the near-IR color maps}

The high-resolution $J-H, J-K$, and $H-K$ color images in Fig. 5 observed in March 2001 confirm our interpretation that the bright components $\mathrm{A}, \mathrm{C}$, and $\mathrm{D}$ are, respectively, the cavity and smaller-scale inhomogeneities in the dense shell, not dense clumps of dust as it might appear from the images alone (Figs. 1-3). In fact, all the bright components coincide with the blue areas in the color images, the cavity A corresponding to the bluest spot. This is a natural consequence of lower optical depths along those directions from the star, with the "hot" stellar photons being scattered into the direction of the observer. 
This is illustrated in Fig. 5 by the dashed lines showing the geometry of our model (Paper III). The bluest, optically thinnest spot is located precisely inside the conical cavity. The star, obscured by $\sim 40$ mag of visual circumstellar extinction, is situated in the red area of the color images, as it was also in the previous epochs (Fig. 16 in Paper III).

\subsubsection{Moving dense layer or dust evaporation?}

The apparent motion of the components A, C, and D (Fig. 1) could be attributed either to the real radial expansion of the opaque dense layer with several "holes" in the dense dust formation zone or to a displacement of the dust formation radius due to evaporation of recently formed dust by a hotter environment. Here, we analyze both processes and argue that the temperature-induced displacement of the dust formation zone is acting in IRC +10216 . For simplicity, we consider a single dust formation radius corresponding to the formation of carbon dust (cf. Paper III).

One can explain the observed decreasing brightness of the star by assuming a monotonically increasing mass-loss rate and, hence, higher densities and optical depths of the wind in the dust formation zone. Higher mass loss and continuing condensation of new dust in the wind out of the gas phase increase the temperatures of the outflowing gas and dust due to backwarming. Increasing temperatures affect the location of the inner dust boundary of the envelope via dust sublimation, causing its displacement with a velocity that has nothing to do with the outflow motion of the envelope.

Crucial to distinguishing between the real motion of a dense layer and the temperature-induced shift of the dust formation zone are estimates of the apparent velocities of the components $\mathrm{A}, \mathrm{C}$, and $\mathrm{D}$ relative to the star. For the assumed distance of $130 \mathrm{pc}$, the linear fit in Fig. 4 gives for the brightest component A a velocity $v_{\mathrm{A}} \approx 18 \mathrm{~km} \mathrm{~s}^{-1}$ in the plane of sky. On the basis of our model (Paper III), one can derive for the component a deprojected radial velocity $v_{\mathrm{rA}} \approx 19 \mathrm{~km} \mathrm{~s}^{-1}$.

Since the deprojected radial velocity is higher than the observed (terminal) wind outflow speed in IRC +10216 of $v \approx$ $15 \mathrm{~km} \mathrm{~s}^{-1}$, it is unlikely that the observed changes reflect just an expansion of a dense layer in which grains are forming. In fact, the standard picture of a stationary stellar wind predicts an acceleration of dust and gas within distances by a factor of 2-5 larger than the dust formation radius (Steffen et al. 1997). Due to the radiation pressure on dust grains, the wind velocity increases in this transition zone, approaching asymptotically the terminal velocity at larger distances. As our model (Paper III) associates the observed components of IRC +10216 with the dust formation zone, we expect that dust and gas are not yet fully accelerated there, i.e. that the radial outflow velocity $v_{\mathrm{r}}<15 \mathrm{~km} \mathrm{~s}^{-1}$.

However, the deprojected velocity $v_{\mathrm{rA}} \approx 19 \mathrm{~km} \mathrm{~s}^{-1}$ is significantly larger than the expected wind velocity in the dust formation zone. Only if we assume an unlikely lower limit of $100 \mathrm{pc}$ (Becklin et al. 1969), does the deprojected velocity $v_{\mathrm{rA}}$ approach (from above) $v \approx 15 \mathrm{~km} \mathrm{~s}^{-1}$, which is still too high for the dust formation zone. If, however, the actual distance to IRC +10216 is larger than $130 \mathrm{pc}$, one would obtain $v_{\mathrm{r}} \gtrsim 19 \mathrm{~km} \mathrm{~s}^{-1}$ and an even larger discrepancy.

Moreover, there are reasons to believe that the acceleration depicted by the parabolic fit in Fig. 4 is real and that the actual apparent motion of $\mathrm{A}$ is now as fast as $v_{\mathrm{A}} \approx 26 \mathrm{~km} \mathrm{~s}^{-1}$. The corresponding deprojected velocity is then $v_{\mathrm{rA}} \approx 28 \mathrm{~km} \mathrm{~s}^{-1}$, much higher than the expected wind speed, for any realistic distance to IRC +10216 . Taken in context with increasing optical depths in the shell, this suggests that the observed motions are caused by the rapid dust evaporation due to backwarming and higher temperatures in the dense environment formed by the increased mass loss.

We believe that a reasonable interpretation of the observed changes in IRC +10216 would be the following picture. During the recent period of increasing mass-loss which started $\sim 20$ 30 years ago, a compact dense shell has formed around the star. The mass-loss rate was recently as high as $\dot{M} \approx 10^{-4} M_{\odot} \mathrm{yr}^{-1}$ (Paper III) and the innermost regions of the dust shell are expanding outward at a local wind velocity $v \lessgtr 10 \mathrm{~km} \mathrm{~s}^{-1}$. Dust formation continues in the expanding material, thus increasing its optical depth and obscuring the central star. The optical depths in the polar regions remain significantly smaller than in the other regions of the dense shell, making the cavity (A) and the other components relatively brighter than at previous epochs. As the dense, increasingly optically thick dusty shell expands, steeply rising temperatures inside it (due to the backwarming from the steepening density front) inhibit further dust condensation and evaporate outflowing grains. In effect, these processes have been shifting recently the dust formation radius outward with an average velocity $v_{\mathrm{r}} \approx 19 \mathrm{~km} \mathrm{~s}^{-1}$ (or as high as $\sim 30 \mathrm{~km} \mathrm{~s}^{-1}$ in 2001, if the apparent acceleration measured in this work is real). One can predict that the star will remain obscured until $\dot{M}$ starts to drop back to lower values. In a few years from that moment, we could probably be witnessing the star (B) reappearing whereas the cavities becoming relatively fainter.

\subsection{Dust formation models and the fading of $B$}

If the star is located at the position of component $\mathrm{B}$, as is suggested by the two-dimensional radiative transfer model (see Sect. 3.2 and Paper III), the fading of B might be caused by the formation of new dust along the line of sight towards the star. To investigate, whether such a scenario would be capable of explaining the observed time scale of the fading of B, we constructed a very simple gas box model. The idea is to follow the process of carbon grain formation in a gas element moving away from the star at a constant velocity. To characterize the changing thermodynamic conditions experienced by the gas element, we assume a power-law gas temperature stratification

$T^{\mathrm{g}}(r)=T_{0}^{\mathrm{g}}\left(\frac{R_{0}}{r}\right)^{\alpha}$

and evaluate the gas density structure from mass conservation in a stationary, spherically symmetric configuration

$\rho^{\mathrm{g}}(r)=\frac{\dot{M}}{4 \pi v} \frac{1}{r^{2}}=\rho_{0}^{\mathrm{g}}\left(\frac{R_{0}}{r}\right)^{2}$. 
For the co-moving gas element, the time coordinate is given by

$\mathrm{d} t=\frac{\mathrm{d} r}{v}$.

In this gas element, we consider heteromolecular formation and growth of carbon grains which we compute according to the moment method derived by Gail \& Sedlmayr (1988). The growth process includes reactions with the molecular species $\mathrm{C}, \mathrm{C}_{2}, \mathrm{C}_{2} \mathrm{H}$, and $\mathrm{C}_{2} \mathrm{H}_{2}$. In order to calculate the concentrations of the relevant carbon-bearing molecules, chemical equilibrium in the gas phase is assumed. We consider a carbonrich element mixture with otherwise solar abundances.

In Fig. 6 the resulting temporal evolution of the gas box is depicted for the following parameters: $T_{0}=3000 \mathrm{~K}$ at a radius $R_{0}=3.15 \times 10^{13} \mathrm{~cm}$ (corresponding to the Stefan-Boltzmann radius for $T_{0}$ and a stellar luminosity of $15000 L_{\odot}$ ), $\alpha=0.5$ (i.e. an optically thin temperature distribution), $\dot{M}=10^{-4} M_{\odot} \mathrm{yr}^{-1}$ (reflecting a presently increased massloss rate of IRC +10216$)$, a constant outflow velocity of $v=$ $15 \mathrm{~km} \mathrm{~s}^{-1}$, and a carbon abundance (by number) of $\epsilon_{\mathrm{C}}=1.5 \epsilon_{\mathrm{O}}$.

In the upper panel of Fig. 6 the prescribed time evolution of gas temperature (Eq. (1)) and density (Eq. (2)) in the gas box are shown. The second panel depicts the nucleation rate $J_{*}$, i.e. the number of seed particles formed per second and hydrogen nucleus (solid line) and the number of dust grains per hydrogen nucleus (dash-dotted line). In the third panel, the degree of condensation, i.e. the fraction of condensible material that is actually condensed is shown (solid line). The dash-dotted line indicates the optical depth at $2.2 \mu \mathrm{m}$ (measured from the star), that would build up in a stationary situation. To calculate the dust extinction coefficient, we assume the small particle limit of Mie theory. This is justified, since the mean particle radius $\langle a\rangle$ reached after $15 P$ is $5 \times 10^{-2} \mu \mathrm{m}$, giving a maximum size parameter $x=2 \pi\langle a\rangle / \lambda=0.14$ at the considered wavelength of $\lambda=2.2 \mu \mathrm{m}$. The optical properties of the grains are described by the complex refractive index of amorphous carbon tabulated in Preibisch et al. (1993). In the lower panel we plot the normalized intensity at $2.2 \mu \mathrm{m}$ that would emerge along the line of sight from the increasingly obscured radiation source (solid line). This intensity is calculated from the formal solution of the radiative transfer equation:

$\frac{I}{I_{0}}=\mathrm{e}^{-\tau_{2.2 \mu \mathrm{m}}}+\int_{0}^{\tau_{2.2} \mu \mathrm{m}} \frac{B\left(T\left(t^{\prime}\right)\right)}{I_{0}} \mathrm{e}^{-\left(\tau_{\left.2.2 \mu \mathrm{m}-t^{\prime}\right)}\right.} \mathrm{d} t^{\prime}$.

Here we assume thermal emission of the dust grains ( $B$ is the Kirchhoff-Planck function) and normalize the intensity to $I_{0}=$ $B\left(T_{0}\right)$.

For comparison, we also plotted in the lower panel of Fig. 6 the observed intensity of component B, normalized to its intensity in our 1997 speckle image (crosses). The intensity of component $\mathrm{B}$ fades from its maximum value to $17 \%$ of this value within about $1400 \mathrm{~d}$, corresponding to 2.2 pulsation periods of IRC +10216 . This time interval is indicated in the lower panel of Fig. 6 by the vertical dashed lines. The comparison shows that dust condensation in front of the star in fact can reproduce the observed time scale of the fading of component B for realistic values of the parameters characterizing our simple toy model.
In Table 2 the timescale $\tau$ of the intensity drop to $17 \%$ of the initial value is summarized for various parameter combinations. We note a steep dependence of $\tau$ on the $\mathrm{C} / \mathrm{O}$ ratio, i.e. on the amount of material available for dust formation. Consistent model calculations for dust driven outflows (e.g., Winters et al. 2000b; Arndt et al. 1997; Dominik et al. 1990) show, that the outflow velocity of a carbon-rich dust-driven wind also depends strongly on the $\mathrm{C} / \mathrm{O}$ ratio. For the observed outflow velocity of IRC +10216 of $\sim 15 \mathrm{~km} \mathrm{~s}^{-1}$, C/O ratios in the range $(1.20 \lesssim \mathrm{C} / \mathrm{O} \lesssim 1.60)$ are required. Therefore, Table 2 implies, that the present day mass-loss rate of IRC +10216 in fact should have increased considerably above the "canonical" value of (a few) $10^{-5} M_{\odot} / \mathrm{yr}$ derived from CO rotational line observations probing the outer and therefore, older parts of the circumstellar shell (e.g., Schöier \& Olofsson 2001) or from $\mathrm{CO}$ infrared line profiles observed about one decade ago (e.g., Winters et al. 2000a).

Once dust has formed in the circumstellar shell, its opacity would lead to a pronounced backwarming and therefore, to a steeper temperature gradient in the dust formation zone. Accordingly, in our simple gas box model, lower temperatures would be reached at the same density. As a result, dust growth becomes more efficient in this region and a reduced $\mathrm{C} / \mathrm{O}$ ratio is sufficient to recover the observed fading time scale (see Table 2; compare the corresponding entries for $\alpha=0.5$ with $\alpha=0.6$ and 0.7 ).

A more realistic treatment of the dust formation and massloss process in IRC +10216 would require the simultaneous solution of the time dependent hydrodynamic equations, taking into account the pulsation of the star, together with the equations describing the dust formation process and the radiative transfer problem. Such a consistent investigation will be presented in a forthcoming paper.

An alternative interpretation of the fading of component B, together with its increasing separation from $\mathrm{A}$, could be based on a scenario, where the star is located in the direction of component A, as argued in previous studies by Weigelt et al. (1998), Haniff \& Buscher (1998), and Tuthill et al. (2000). In this case, component B would indicate thermal emission from a dust cloud, ejected from the star in a direction almost perpendicular to the line of sight. The temporal evolution of the brightness of B would then be the result of a competition between the initially increasing thermal emission due to ongoing dust formation in the cloud and decreasing temperature of the cloud as it is moving away from the star. Preliminary results for this scenario, which are based on a consistent model calculation, are presented in Winters et al. (2002). Although the time scale of the fading of component B is reproduced quite well in this model, the calculated intensity ratio between the expanding cloud and the star is smaller than the observed intensity ratio between components $\mathrm{B}$ and $\mathrm{A}$ by about a factor of 3 .

\section{Summary}

We presented new near-infrared $(J H K)$ bispectrum speckleinterferometry monitoring of the carbon star IRC +10216 obtained during 1999-2001 with the SAO $6 \mathrm{~m}$ telescope. The $J$-, $H$-, and $K$-band resolutions are 50 mas, 56 mas, and 73 mas, 


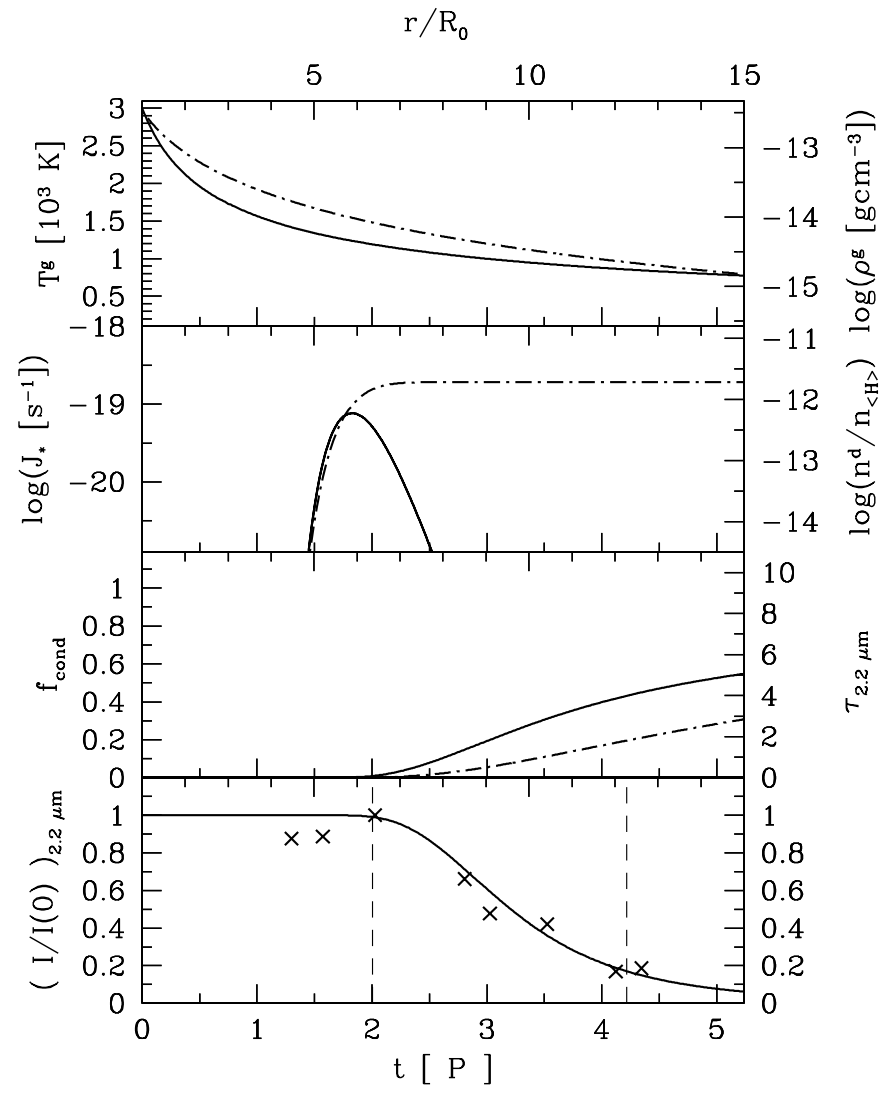

Fig. 6. Temporal evolution of the gas box. The time coordinate is given in units of $P=649 \mathrm{~d}$, the pulsation period of IRC +10216 . Upper panel: gas temperature (Eq. (1), solid line, 1.h.s. ordinate) and density (Eq. (2), dash-dotted line, r.h.s. ordinate); second panel: dust nucleation rate $J_{*}$ (solid line, 1.h.s. ordinate) and number of dust grains per hydrogen nucleus (dash-dotted line, r.h.s. ordinate); third panel: degree of condensation $f_{\text {cond }}$ (solid line, 1.h.s. ordinate) and optical depth at $2.2 \mu \mathrm{m}$ (dash-dotted line, r.h.s. ordinate); lower panel: normalized emergent intensity at $2.2 \mu \mathrm{m}$ (solid line) and observed intensity of component B, normalized to its maximum value (crosses). See text for more details.

respectively. Together with the data of Paper II, the available $K$ band observations cover now 8 epochs from 1995 to 2001 and show the dynamic evolution of the inner dust shell. Our images show very good agreement with the images (1997-1999) reported by Tuthill et al. (2000). Four main components within a 0.2 radius can be identified, which are surrounded by a fainter asymmetric nebula. The apparent separation of the two initially brightest components A and B increased from 191 mas in 1995 to 351 mas in 2001. At the same time, component B has been fading and almost disappeared in 2000 whereas the initially faint components $\mathrm{C}$ and $\mathrm{D}$ became brighter. There is weak evidence for an accelerated apparent motion of component $\mathrm{B}$ with respect to $\mathrm{A}$. This might be related to the beginning development of a fast polar wind or, as favored by the radiative transfer calculations, to rapid dust evaporation due to backwarming effects. The changes of the images can be related to changes of the optical depth caused, for example, by mass-loss variations or new dust condensation in the wind. The observed relative motion of components $\mathrm{A}$ and $\mathrm{B}$ with a deprojected
Table 2. Parameters of the gas box model and resulting fading time scales for an assumed outflow velocity of $v=15 \mathrm{~km} \mathrm{~s}^{-1}$.

\begin{tabular}{cccccc}
\hline \hline$\alpha$ & $\begin{array}{c}T_{0} \\
\mathrm{~K}\end{array}$ & $\begin{array}{c}\rho_{0} \\
10^{-13} \mathrm{~g} / \mathrm{cm}^{3}\end{array}$ & $\begin{array}{c}\dot{M} \\
M_{\odot} / \mathrm{yr}\end{array}$ & $\mathrm{C} / \mathrm{O}$ & $\begin{array}{c}\tau \\
649 \mathrm{~d}\end{array}$ \\
\hline 0.5 & 2800 & 2.95 & $10^{-4}$ & 1.5 & 1.83 \\
0.5 & 2800 & 2.95 & $10^{-4}$ & 1.4 & $>13$ \\
0.5 & 2800 & 2.95 & $10^{-4}$ & 1.3 & $\rightarrow \infty$ \\
0.5 & 2500 & 4.68 & $10^{-4}$ & 1.5 & 0.65 \\
0.5 & 2500 & 4.68 & $10^{-4}$ & 1.4 & 1.79 \\
0.5 & 2500 & 4.68 & $10^{-4}$ & 1.3 & $\rightarrow \infty$ \\
0.5 & 2500 & 0.47 & $10^{-5}$ & 2.1 & 1.58 \\
0.5 & 2500 & 0.47 & $10^{-5}$ & 2.0 & 3.05 \\
0.5 & 2500 & 0.47 & $10^{-5}$ & 1.9 & $\rightarrow \infty$ \\
0.5 & 3000 & 3.37 & $10^{-4}$ & 1.6 & 1.04 \\
0.5 & 3000 & 3.37 & $10^{-4}$ & 1.5 & 2.22 \\
0.5 & 3000 & 3.37 & $10^{-4}$ & 1.4 & $\rightarrow \infty$ \\
0.4 & 3000 & 3.37 & $10^{-4}$ & 1.6 & 2.59 \\
0.4 & 3000 & 3.37 & $10^{-4}$ & 1.5 & 7.53 \\
0.6 & 3000 & 3.37 & $10^{-4}$ & 1.5 & 1.15 \\
0.6 & 3000 & 3.37 & $10^{-4}$ & 1.4 & 5.01 \\
0.7 & 3000 & 3.37 & $10^{-4}$ & 1.5 & 0.75 \\
0.7 & 3000 & 3.37 & $10^{-4}$ & 1.4 & 2.54 \\
0.7 & 3000 & 0.34 & $10^{-5}$ & 2.1 & 2.35 \\
0.7 & 3000 & 0.34 & $10^{-5}$ & 1.9 & 9.62 \\
0.7 & 3000 & 0.34 & $10^{-5}$ & 1.5 & $\rightarrow \infty$ \\
\hline & & & & &
\end{tabular}

velocity of $19 \mathrm{~km} \mathrm{~s}^{-1}$ is most likely due to dust evaporation in the optically thicker and hotter environment.

The present monitoring covers more than 3 pulsation periods and shows that the structural variations are not related to the stellar pulsation cycle in a simple way. This is consistent with the predictions of hydrodynamical models that enhanced dust formation takes place on a timescale of several pulsation periods. We have demonstrated, that formation of new dust along the line of sight towards the star can explain the observed fading time scale of component $\mathrm{B}$, for reasonable values of the parameters involved in our simple gas box model. In particular, this dust formation calculation lends independent support to the previous finding that the present-day mass loss rate of IRC +10216 should be of the order of $10^{-4} M_{\odot} / y r$. Further high-resolution observations will be most important for testing different views and models, and for better understanding of the evolution of this complicated nebula.

Acknowledgements. The observations were made with the SAO $6 \mathrm{~m}$ telescope, operated by the Special Astrophysical Observatory, Russia. We thank Boris Yudin for providing near-infrared photometric data of IRC +10216 . This research has made use of the SIMBAD database, operated by CDS in Strasbourg, and of NASA's Astrophysics Data System.

\section{References}

Arndt, T. U., Fleischer, A. J., \& Sedlmayr, E. 1997, A\&A, 327, 614

Balick, B. 1987, AJ, 94, 671 
Becklin, E. E., Frogel, J. A., Hyland, A. R., Kristian, J., \& Neugebauer, G. 1969, ApJ, 158, L133

Blöcker, T. 1999, Asymptotic Giant Branch Stars, IAU Symp. 191, ed. T. Le Bertre, A. Lebre, \& C. Waelkens, Astron. Soc. Pac., 21

Crosas, M., \& Menten, K. 1997, ApJ, 483, 913

Dominik, C., Gail, H.-P., Sedlmayr, E., \& Winters, J. M. 1990, A\&A, 240,365

Fleischer, A. J., Gauger, A., \& Sedlmayr, E. 1995, A\&A, 297, 543

Gail, H.-P., \& Sedlmayr, E. 1988, A\&A, 206, 153

Gauger, A., Balega, Y., Irrgang, P., Osterbart, R., \& Weigelt, G. 1999, A\&A, 346, 509

Groenewegen, M. A. T., van der Veen, W. E. C. J., \& Matthews, H. E. 1998, A\&A, 338, 491

Haniff, C. A., \& Buscher, D. F. 1998, A\&A, 334, L5

Hofmann, K.-H., \& Weigelt, G. 1986, A\&A, 167, L15

Hofmann, K.-H., Balega, Y., Blöcker, T., \& Weigelt, G. 2001, A\&A, 379,529

IRAS Catalog of Point Sourcesb 1986, Version 2.0, Joint IRAS Science W. G.

Kwok, S. 1982, ApJ, 258, 280

Labeyrie, A. 1970, A\&A, 6, 85

Le Bertre, T. 1992, A\&AS, 94, 377

Lohmann, A. W., Weigelt, G., \& Wirnitzer, B. 1983, Appl. Opt., 22, 4028

Loup, C., Forveille, T., Omont, A., \& Paul, J. F. 1993, A\&AS, 99, 291

Mauron, N., \& Huggins, P. J. 1999, A\&A, 349, 203

Mauron, N., \& Huggins, P. J. 2000, A\&A, 359, 707
Men'shchikov, A. B., Balega, Y. Y., Blöcker, T., Osterbart, R., \& Weigelt, G. 2001, A\&A, 368, 497 (Paper III)

Monnier, J. D., Tuthill, P. G., \& Danchi, W. C. 2000, ApJ, 545, 957

Olofsson, H. 1996, ApSS, 245, 169

Osterbart, R., Balega, Y., Blöcker, T., Men'shchikov, A. B., \& Weigelt, G. 2000, A\&A, 357, 169 (Paper II)

Preibisch, T., Ossenkopf, V., Yorke, H. W., \& Henning, T. 1993, A\&A, 279,577

Schöier, F. L., \& Olofsson, H. 2001, A\&A, 368, 969

Steffen, M., Szczerba, R., Men'shchikov, A. B., \& Schönberner, D. 1997, A\&AS, 126, 39

Tuthill, P. G., Monnier, J. D., Danchi, W. C., \& Lopez, B. 2000, ApJ, 543,284

Weigelt, G. 1977, Opt. Commun., 21, 55

Weigelt, G., Balega, Y., Hofmann, K.-H., Langer, N., \& Osterbart, R. 1997, Science with the VLT Interferometer, ESO Astrophysics Symposia, 206

Weigelt, G., Balega, Y., Blöcker, T., et al. 1998, A\&A, 333, L51 (Paper I)

Winters, J. M., Blöcker, T., Hofmann, K.-H., \& Weigelt, G. 2002, in IAU Symp. 209, Planetary Nebulae and Their Role in the Universe, ed. M. Dopita, S. Kwok, \& R. S. Sutherland, ASP, in press

Winters, J. M., Keady, J. J., Gauger, A., \& Sada, P. V. 2000a, A\&A, 359,651

Winters, J. M., Le Bertre, T., Jeong, K. S., Helling, C., \& Sedlmayr, E. 2000b, A\&A, 361, 641 